\title{
Some New Coincidence Theorems in Product GFC-Spaces with Applications
}

\author{
Jianrong Zhao \\ School of Economic Mathematics, Southwestern University of Finance and Economics, Chengdu 610074, China \\ Correspondence should be addressed to Jianrong Zhao; mathzjr@swufe.edu.cn
}

Received 28 February 2014; Accepted 12 April 2014; Published 6 May 2014

Academic Editor: Xie-ping Ding

Copyright (C) 2014 Jianrong Zhao. This is an open access article distributed under the Creative Commons Attribution License, which permits unrestricted use, distribution, and reproduction in any medium, provided the original work is properly cited.

We first propose a new concept of GFC-subspace. Using this notion, we obtain a new continuous selection theorem. As a consequence, we establish some new collective fixed point theorems and coincidence theorems in product GFC-spaces. Finally, we give some applications of our theorems.

\section{Introduction and Preliminaries}

Recently, Ding [1, 2], X. P. Ding and T. M. Ding [3], Tang et al. [4], Fang and Huang [5] established some collective fixed point theorems, coincidence theorems, and KKM-type theorems for the families of set-valued mappings, which are defined on FC-spaces or product FC-spaces without any convexity structure.

Very recently, Khanh and Quan [6], Khanh et al. [7] defined a new notion by GFC-space which is a generalization of FC-space and proved some continuous selections theorems, collectively fixed point theorems, coincidence theorems, and KKM-type theorems in this new GFC-space.

Motivated and inspired by the work mentioned above, we will give some new collective fixed point and coincidence theorems in product GFC-spaces in the present paper. For this purpose, we first propose a new concept of GFCsubspace. Using this notion, we obtain a new continuous selection theorem. As a consequence, we establish some new collective fixed point theorems and coincidence theorems in product GFC-spaces. Finally, we give some applications of our theorems.

For our purpose, first, we present some known definitions and preliminary results. For a nonempty set $A,\langle A\rangle$ denotes the family of all nonempty finite subsets and $2^{A}$ denotes the family of all subsets of $A$. We denote the standard $n$ simplex with vertices $\left\{e_{i}\right\}_{i=0}^{n}$ by $\Delta_{n}$. The following notion was introduced by Khanh and Quan [6].
Definition 1 (see [6]). (a) A generalized finitely continuous topological space or a GFC-space $\left(X, Y,\left\{\varphi_{N}\right\}\right)$ consists of a topological space $X$ and a nonempty set $Y$ such that for each finite subset $N=\left\{y_{0}, y_{1}, \ldots, y_{n}\right\} \in\langle Y\rangle$, one has a continuous mapping $\varphi_{N}: \Delta_{n} \rightarrow X$.

(b) Let $A, B \subset Y$ and $S: Y \rightarrow 2^{X}$ be given. Then $B$ is called an $S$-subset of $Y$ with respect to $A$ if for any $N=$ $\left\{y_{0}, y_{1}, \ldots, y_{n}\right\} \in\langle Y\rangle$ and for any $\left\{y_{i 0}, \ldots, y_{i k}\right\} \subset A \cap N$, one has $\varphi_{N}\left(\Delta_{k}\right) \subset S(B)$, where $\Delta_{k}=\operatorname{co}\left(\left\{e_{i 0}, \ldots, e_{i k}\right\}\right)$.

Now we give a new concept as follows.

Definition 2. Assume that $\left(X, Y,\left\{\varphi_{N}\right\}\right)$ is a GFC-space, $C \subset Y$, and $D \subset X$. Then $D$ is said to be a GFC-subspace of $X$ with respect to $C$ if for each $N=\left\{y_{0}, y_{1}, \ldots, y_{n}\right\} \in\langle Y\rangle$ and for any $\left\{y_{i 0}, \ldots, y_{i k}\right\} \subset C \cap N$, one has $\varphi_{N}\left(\Delta_{k}\right) \subset D$, where $\Delta_{k}=$ $\operatorname{co}\left(\left\{e_{i 0}, \ldots, e_{i k}\right\}\right)$.

Remark 3. By the Definition 2, we know that $\left(D, C,\left\{\varphi_{N}\right\}\right)$ is also a GFC-space. Clearly, if $B$ is an $S$-subset of $Y$ with respect to $A$, then $S(B)$ is a GFC-subspace of $X$ with respect to $A$. In addition, if $X=Y$, then a GFC-subspace $D$ of $X$ with respect to $C$ coincides with an FC-subspace $D$ of $X$ with respect to $C$ (see [1]).

Assume that $\left\{D_{i}\right\}_{i \in I}$ is a family of GFC-subspace of $X$ with respect to $C$ and $\bigcap_{i \in I} D_{i} \neq \emptyset$, where $I$ is an index set. It then follows from Definition 2 that $\bigcap_{i \in I} D_{i}$ is also a GFC-subspace 
of $X$ with respect to $C$. For any given subset $A$ of $X$, we define GFC-hull of $A$ with respect to $C$ by

$$
\begin{aligned}
& \operatorname{GFC}(A, C) \\
& =\cap\{B \subset X: A \subset B, \\
& \quad B \text { is a GFC-subspace of } X \text { with respect to } C\} .
\end{aligned}
$$

We simply write $\mathrm{GFC}(S(C))$ instead of $\mathrm{GFC}(S(C), C)$ when $A=S(C)$. We can easily show that $\operatorname{GFC}(A, C)$ is also a GFC-subspace of $X$ with respect to $C$.

Let $X$ be a topological space. A set $A \subset X$ is called compactly closed (resp., compactly open) if for each nonempty compact set $K \subset X$ such that $A \cap K$ is closed (resp., open) in $K$. And the compact interior and the compact closure of $A$ (see [8]) are defined by

$$
\begin{aligned}
& \operatorname{cint}(A)=\cup\{B \subset X: B \text { is compactly open in } X, B \subset A\}, \\
& \operatorname{ccl}(A)=\cap\{B \subset X: B \text { is compactly closed in } X, B \subset A\} .
\end{aligned}
$$

Clearly if $K$ is a nonempty compact subset of $X$, then we deriver that $\operatorname{cint}(A) \cap K=\operatorname{int}_{K}(A \cap K), \operatorname{ccl}(A) \cap K=\mathrm{cl}_{K}(A \cap$ $K)$, and $\operatorname{ccl}(A \backslash K)=X \backslash \operatorname{cint}(A)$.

Assume that $Y$ and $Z$ are nonempty sets and $X$ is a topological space. Define two set-valued mappings $F: X \times$ $Y \rightarrow 2^{Z}$ and $C: X \rightarrow 2^{Z} . F(x, y)$ is called transfer compactly upper semicontinuous in $x$ with respect to $C$ (see [9]) if for any nonempty compact subset $K$ of $X$ and any $x \in K$, the set $\{y \in Y: F(x, y) \in C(x)\} \neq \emptyset$ means that there is a relatively open neighborhood $N(x)$ of $x$ in $K$ and a point $y^{\prime} \in Y$ such that $F\left(z, y^{\prime}\right) \subset C(z)$ for all $z \in N(x)$.

A mapping $T: X \rightarrow 2^{Y}$ is called transfer compactly open-valued (resp., transfer compactly closed-valued) on $X$ (see [8]) if for each $x \in X$ and each nonempty compact subset $K$ of $Y, y \in T(x) \cap K$ (resp., $y \notin T(x) \cap K$ ) means that there is $x^{\prime} \in X$ such that $y \in \operatorname{int}_{K}\left(T\left(x^{\prime}\right) \cap K\right)$ (resp., $\left.y \notin \mathrm{cl}_{K}\left(T\left(x^{\prime}\right) \cap K\right)\right)$.

We need the following two results. The first statement was proved by Ding and Park [9].

Lemma 4 (see [9]). Let $Y$ and $Z$ be two nonempty sets and $X a$ topological space. Let $F: X \times Y \rightarrow 2^{Z}$ and $C: X \rightarrow 2^{Z}$ be two set-valued mappings. Then $F(x, y)$ is transfer compactly upper semicontinuous in $x$ with respect to $C$ if and only if the mapping $T: Y \rightarrow 2^{X}$ defined by $T(y)=\{x \in X: F(x, y) \not \subset C(x)\}$ is transfer compactly closed-valued on $Y$.

The second statement is Lemma 1.1 of Ding [8].

Lemma 5. Assume that $X$ and $Y$ are two topological spaces and $F: X \rightarrow 2^{Y}$ is a set-valued mapping with nonempty values. Then the following statements are equivalent:

(i) $F^{-1}: Y \rightarrow 2^{X}$ is transfer compactly open-valued;

(ii) for any compact subset $K$ of $X$ and any $x \in K$, one has $y \in Y$ satisfying that $x \in \operatorname{cint} F^{-1}(y) \cap K$ and $K=\bigcup_{y \in Y} \operatorname{int}_{K}\left(F^{-1} \cap K\right)$.
Throughout this paper, we always let $I$ and $J$ be any given index set. Now we give the following statement, which generalizes Lemma 1.1 of Ding [1].

Lemma 6. Suppose that $\left(X_{i}, Y_{i},\left\{\varphi_{N_{i}}\right\}\right)$ is a GFC-space for each $i \in$ I. If $X=\prod_{i \in I} X_{i}, Y=\prod_{i \in I} Y_{i}$, and $\varphi_{N}=\prod_{i \in I} \varphi_{N_{i}}$, then $\left(X, Y,\left\{\varphi_{N}\right\}\right)$ is also a GFC-space.

Proof. Let $\pi_{i}: Y \rightarrow Y_{i}$ be the projective mapping from $Y$ to $Y_{i}$ for each $i \in I$. For any given $N=\left\{y_{0}, y_{1}, \ldots, y_{n}\right\} \in\langle Y\rangle$, we denote $N_{i}=\pi_{i}(N)=\left\{\pi_{i}\left(y_{0}\right), \ldots, \pi_{i}\left(y_{n}\right)\right\} \in\left\langle Y_{i}\right\rangle$. Note that $\left(X_{i}, Y_{i},\left\{\varphi_{N_{i}}\right\}\right)$ is a GFC-space. Then we have a continuous mapping $\varphi_{N_{i}}: \Delta_{n} \rightarrow X_{i}$ for each $i \in I$. So we may let a mapping $\varphi_{N}: \Delta_{n} \rightarrow X$ by $\varphi_{N}(\alpha)=\prod_{i \in I} \varphi_{N_{i}}(\alpha)$, for any $\alpha \epsilon$ $\Delta_{n}$. It follows that $\varphi_{N}$ is a continuous mapping, which means that $\left(X, Y,\left\{\varphi_{N}\right\}\right)$ is a GFC-space. So Lemma 6 is proved.

\section{Continuous Selection and Collective Fixed Points}

Theorem 7. Assume that $\left(X, Y,\left\{\varphi_{N}\right\}\right)$ is a GFC-space and $Z$ is a compact topological space. Suppose that $F: Z \rightarrow 2^{Y}$ and $G: Z \rightarrow 2^{X}$ are such that

(i) $Z=\bigcup_{y \in Y} \operatorname{cint} F^{-1}(y)$;

(ii) for any given $z \in Z, G(z)$ is a GFC-subspace of $X$ with respect to $F(z)$.

Then one has a continuous selection $g: Z \rightarrow X$ of $G$ satisfying $g=\varphi \circ \psi$, where $\varphi: \Delta_{n} \rightarrow X$ and $\psi: Z \rightarrow \Delta_{n}$ are continuous for some $n \in \mathbb{Z}^{+}$.

Proof. By condition (i), we know that there exists $N=$ $\left\{y_{0}, y_{1}, \ldots, y_{n}\right\} \in\langle Y\rangle$ such that $Z=\bigcup_{i=0}^{n} \operatorname{cint} F^{-1}\left(y_{i}\right)$ since $Z$ is compact. Assume that $\left\{\psi_{i}\right\}_{i=0}^{n}$ is the continuous partition of unity subordinated to the open covering $\left\{\operatorname{cint} F^{-1}\left(y_{i}\right)\right\}_{i=0}^{n}$; then for any $i \in\{0,1, \ldots, n\}$ and $z \in Z$, one has

$$
\psi_{i}(z) \neq 0 \Longleftrightarrow z \in \operatorname{cint} F^{-1}\left(y_{i}\right) \subset F^{-1}\left(y_{i}\right) \Longrightarrow y_{i} \in F(z) .
$$

Let $\psi: Z \rightarrow \Delta_{n}$ be a mapping with $\psi(z)=\sum_{i=0}^{n} \psi_{i}(z) e_{i}$. Clearly $\psi$ is continuous and for any $z \in Z$, one has $\psi(z)=$ $\sum_{i \in J(x)} \psi_{j}(z) e_{j}$ where $J(x)=\left\{j \in\{0,1, \ldots, n\}: \psi_{i}(z) \neq 0\right\}$. So by (3), we get $\left\{y_{j}: j \in J(x)\right\} \subset F(z) \cap N$. It then follows from condition (ii) that for $z \in Z, \varphi_{N}\left(\Delta_{J(x)}\right) \subset G(z)$. It is easy to see that $g(z)=\varphi_{N} \circ \psi(z) \in \varphi_{N}\left(\Delta_{J(x)}\right) \subset G(z)$, which implies that $g=\varphi_{N} \circ \psi$ is a continuous selection of $G$. The proof of Theorem 7 is completed.

Remark 8. Applying the definition of GFC-subspace, we extend Theorem 2.2 of Tarafdar [10], Proposition 1 of Browder [11], and Theorem 2.1 of Ding [2] to GFC-spaces without any convexity.

Theorem 9. Assume that $X=\prod_{i \in I} X_{i}$ and $\left(X_{i}, Y_{i},\left\{\varphi_{N_{i}}\right\}\right)$ is a compact GFC-space for each $i \in I$. Suppose that $F_{i}: X \rightarrow 2^{Y_{i}}$ 
and $G_{i}: X \rightarrow 2^{X_{i}}$ are two set-valued mappings satisfying the following conditions:

(i) $X=\bigcup_{y_{i} \in Y_{i}} \operatorname{cint} F_{i}^{-1}\left(y_{i}\right)$;

(ii) for any given $x \in X, G_{i}(x)$ is a GFC-subspace of $X_{i}$ with respect to $F_{i}(x)$.

Then one has a point $\widehat{x}=\left(\widehat{x}_{i}\right)_{i \in I} \in X$ such that $\widehat{x}_{i} \in G_{i}(\widehat{x})$ for $i \in I$.

Proof. For any given $i \in I$, using Theorem 7, we obtain that there are continuous mappings $\varphi_{N_{i}}: \Delta_{n_{i}} \rightarrow X_{i}$ and $\psi_{i}: X \rightarrow \Delta_{n_{i}}$ such that $g_{i}=\varphi_{N_{i}} \circ \psi_{i}$ is a continuous selection of $G_{i}$ for some positive integer $n_{i}$. Assume that $E_{i}$ is the linear hull of the set $\left\{e_{0}, e_{1}, \ldots, e_{n_{i}}\right\}$ for each $i \in I$. Clearly $E_{i}$ is a locally convex Hausdorff topological vector space as it is finite dimensional and $\Delta_{n_{i}}$ is a compact convex subset of $E_{i}$. Moreover, $E=\prod_{i \in I} E_{i}$ is a locally convex Hausdorff topological vector space and $\Delta=\prod_{i \in I} \Delta_{n_{i}}$ is a compact convex subset of $E$. Now let $\Phi: \Delta \rightarrow X$ and $\Psi: X \rightarrow \Delta$ be two continuous mappings with

$$
\Phi(\delta)=\prod_{i \in I} \varphi_{N_{i}}\left(P_{i}(\delta)\right), \quad \Psi(x)=\prod_{i \in I} \psi_{i}(x),
$$

where $P_{i}: \Delta \rightarrow \Delta_{n_{i}}$ is the projection of $\Delta$ onto $\Delta_{n_{i}}$ for any given $i \in I$. It then follows from the Tychonoff fixed point theorem that the continuous mapping $\Psi \circ \Phi: \Delta \rightarrow \Delta$ has a fixed point $\delta \in \Delta$; that is, $\delta=\Psi \circ \Phi(\delta)$. Let $\widehat{x}=\Phi(\delta)$. It follows that

$$
\begin{aligned}
\widehat{x} & =\Psi \circ \Phi(\widehat{x})=\Phi\left(\prod_{i \in I} \psi_{i}(\widehat{x})\right) \\
& =\prod_{i \in I} \varphi_{N_{i}}\left(P_{i}\left(\prod_{i \in I} \psi_{i}(\widehat{x})\right)\right)=\prod_{i \in I} \varphi_{N_{i}} \circ \psi_{i}(\widehat{x}) .
\end{aligned}
$$

This means that $\widehat{x}=\varphi_{N_{i}} \circ \psi_{i}(\hat{x}) \in G_{i}(\hat{x})$ for each $i \in I$. So Theorem 9 is proved.

Theorem 10. Assume that $Z_{i}$ is a topological space and $\left(X_{i}, Y_{i},\left\{\varphi_{N_{i}}\right\}\right)$ is a GFC-space for each $i \in I$. Suppose that $X=\prod_{i \in I} X_{i}, S_{i}: Z_{i} \rightarrow 2^{Y_{i}}$ and $T_{i}: Z_{i} \rightarrow 2^{X_{i}}$ and $g_{i}: X \rightarrow Z_{i}$ is a continuous mapping such that

(i) for any compact subset $D_{i}$ of $Z_{i}, D_{i}=\bigcup_{y_{i} \in Y_{i}}\left(\operatorname{cint} S_{i}^{-1}\right.$ $\left.\left(y_{i}\right) \cap D_{i}\right)$

(ii) for any $z_{i} \in Z_{i}, T_{i}\left(z_{i}\right)$ is a GFC-subspace of $X_{i}$ with respect to $S_{i}\left(z_{i}\right)$;

(iii) there is a nonempty subset of $Y_{i}^{0} \subset Y_{i}$ such that the set $B_{i}=\bigcap_{y_{i} \in Y_{i}^{0}}\left(\operatorname{cint} S_{i}^{-1}\left(y_{i}\right)\right)^{c}$ is empty or compact in $Z_{i}$, and for any $N_{i} \in\left\langle Y_{i}\right\rangle$, there exists a compact GFCsubspace $K_{N_{i}}$ of $X_{i}$ with respect to $L_{N_{i}}$ containing $Y_{i}^{0} U$ $N_{i}$.

Then one has a point $\widehat{x}=\left(\widehat{x}_{i}\right)_{i \in I} \in X$ such that $\widehat{x}_{i} \in T_{i}\left(g_{i}(\widehat{x})\right)$ for each $i \in I$.
Proof. For any given $i \in I$, if $B_{i}$ is a nonempty compact subset of $Z_{i}$, then by (i) one has

$$
B_{i}=\bigcup_{y_{i} \in Y_{i}}\left(\operatorname{cint} S_{i}^{-1}\left(y_{i}\right) \cap B_{i}\right) \subset \bigcup_{y_{i} \in Y_{i}} \operatorname{cint} S_{i}^{-1}\left(y_{i}\right) .
$$

Moreover, noticing that $B_{i}$ is compact, we can find that there is a finite set $N_{i} \in\left\langle Y_{i}\right\rangle$ such that

$$
B_{i}=\bigcap_{y_{i} \in Y_{i}^{0}}\left(\operatorname{cint} S_{i}^{-1}\left(y_{i}\right)\right)^{c} \subset \bigcup_{y_{i} \in N_{i}} \operatorname{cint} S_{i}^{-1}\left(y_{i}\right) .
$$

It then follows from (7) that

$$
Z_{i}=\bigcup_{y_{i} \in Y_{i}^{0}} \operatorname{cint} S_{i}^{-1}\left(y_{i}\right) \cup\left(\bigcup_{y_{i} \in N_{i}} \operatorname{cint} S_{i}^{-1}\left(y_{i}\right)\right) .
$$

If $B_{i}$ is empty in (iii), then we derive that

$$
Z_{i}=Z_{i} \backslash B_{i}=Z_{i} \backslash \bigcap_{y_{i} \in Y_{i}^{0}}\left(\operatorname{cint} S_{i}^{-1}\left(y_{i}\right)\right)^{c}=\bigcup_{y_{i} \in Y_{i}^{0}} \operatorname{cint} S_{i}^{-1}\left(y_{i}\right) .
$$

From condition (iii), we know that there exists a compact GFC-subspace $K_{N_{i}}$ of $X_{i}$ with respect to $L_{N_{i}}$ containing $Y_{i}^{0} \cup$ $N_{i}$. So by (8) and (9), we get

$$
Z_{i}=\bigcup_{y_{i} \in L_{N_{i}}}\left(\operatorname{cint} S_{i}^{-1}\left(y_{i}\right)\right) \text {. }
$$

Now let $K_{N}=\prod_{i \in I} K_{N_{i}}, L_{N}=\prod_{i \in I} L_{N_{i}}$, and $\varphi_{N}=$ $\prod_{i \in I} \varphi_{N_{i}}$. By condition (iii), we deduce that $\left(K_{N_{i}}, L_{N_{i}},\left\{\varphi_{N_{i}}\right\}\right)$ is a compact GFC-space. It then follows from Lemma 6 that $\left(K_{N}, L_{N},\left\{\varphi_{N}\right\}\right)$ is also a compact GFC-space. Let $P_{i}: K_{N} \rightarrow$ $2^{L_{N_{i}}}$ and $Q_{i}: K_{N} \rightarrow 2^{K_{N_{i}}}$ be two set-valued mappings with

$$
\begin{aligned}
P_{i}(x) & =S_{i}\left(g_{i}(x)\right) \cap L_{N_{i}}, \\
Q_{i}(x) & =T_{i}\left(g_{i}(x)\right) \cap K_{N_{i}} \text { for } x \in K_{N} .
\end{aligned}
$$

In order to show that the conditions (i) and (ii) of Theorem 9 hold, we only need to show that $Q_{i}(x)$ is a compact GFC-subspace of $K_{N_{i}}$ with respect to $P_{i}(x)$, and $K_{N}=$ $\bigcup_{y_{i} \in L_{N_{i}}} \operatorname{cint} P_{i}^{-1}\left(y_{i}\right)$. By conditions (ii) and (iii), it is easy to see that $Q_{i}(x)$ is a compact GFC-subspace of $K_{N_{i}}$ with respect to $P_{i}(x)$. It remains to show that $K_{N}=\bigcup_{y_{i} \in L_{N_{i}}} \operatorname{cint} P_{i}^{-1}\left(y_{i}\right)$. On one hand, for each $y_{i} \in L_{N_{i}}$, we deduce that

$$
\begin{aligned}
P_{i}^{-1}\left(y_{i}\right) & \\
& =\left\{x \in K_{N}: y_{i} \in P_{i}(x)\right\} \\
& =\left\{x \in K_{N}: y_{i} \in S_{i}\left(g_{i}(x)\right) \cap L_{N_{i}}\right\} \\
& =\left\{x \in K_{N}: y_{i} \in S_{i}\left(g_{i}(x)\right)\right\} \\
& =\left\{x \in K_{N}: x \in g_{i}^{-1}\left(S_{i}^{-1}\left(y_{i}\right)\right)\right\} \\
& =g_{i}^{-1}\left(S_{i}^{-1}\left(y_{i}\right)\right) .
\end{aligned}
$$


On the other hand, by (10), we obtain

$$
g_{i}\left(K_{N}\right) \subset Z_{i}=\bigcup_{y_{i} \in L_{N_{i}}}\left(\operatorname{cint} S_{i}^{-1}\left(y_{i}\right)\right) .
$$

It then follows form (12), (13), and the continuity of $g_{i}$ that

$$
\begin{aligned}
K_{N} & \subset g_{i}^{-1}\left(\bigcup_{y_{i} \in L_{N_{i}}} \operatorname{cint} S_{i}^{-1}\left(y_{i}\right)\right) \\
& =\bigcup_{y_{i} \in L_{N_{i}}} g_{i}^{-1}\left(\operatorname{cint} S_{i}^{-1}\left(y_{i}\right)\right) \\
& =\bigcup_{y_{i} \in L_{N_{i}}}\left(\operatorname{cint} P_{i}^{-1}\left(y_{i}\right)\right) \subset K_{N} .
\end{aligned}
$$

Hence $K_{N}=\bigcup_{y_{i} \in L_{N_{i}}} \operatorname{cint} P_{i}^{-1}\left(y_{i}\right)$. Then Theorem 9 tells us that there exists a point $\widehat{x} \in K_{N} \subset X$ such that $\widehat{x}_{i} \in Q_{i}(\widehat{x})=$ $T_{i}\left(g_{i}(\widehat{x})\right) \cap K_{N_{i}} \subset T_{i}\left(g_{i}(\widehat{x})\right)$ for each $i \in I$. This completes the proof of Theorem 10 .

Theorem 11. Let $X=\prod_{i \in I} X_{i}, Z_{i}$ be a topological space and $\left(X_{i}, Y_{i},\left\{\varphi_{N_{i}}\right\}\right)$ a GFC-space for each $i \in I$. Assume that $S_{i}:$ $Z_{i} \rightarrow 2^{Y_{i}}, T_{i}: Z_{i} \rightarrow 2^{X_{i}}$, and $R_{i}: Y_{i} \rightarrow 2^{X_{i}}$ are set-valued mappings and $g_{i}: X \rightarrow Z_{i}$ is a continuous mapping such that

(i) for any compact subset $D_{i}$ of $Z_{i}, D_{i}=\bigcup_{y_{i} \in Y_{i}}\left(\operatorname{cint} S_{i}^{-1}\right.$ $\left.\left(y_{i}\right) \cap D_{i}\right)$;

(ii) for any $z_{i} \in Z_{i}, \operatorname{GFC}\left(R_{i}\left(S_{i}\left(z_{i}\right)\right)\right) \subset T_{i}\left(z_{i}\right)$;

(iii) if $Z_{i}$ is not compact, then there is a nonempty subset of $Y_{i}^{0} \subset Y_{i}$ such that a compact subset $D_{i}$ of $Z_{i}$ satisfying $Z_{i} \backslash D_{i} \subset \bigcup_{y_{i} \in Y_{i}^{0}} \operatorname{cint} S_{i}^{-1}\left(y_{i}\right)$, and for any $N_{i} \in\left\langle Y_{i}\right\rangle$, there exists a compact GFC-subspace $K_{N_{i}}$ of $X_{i}$ with respect to $L_{N_{i}}$ containing $Y_{i}^{0} \cup N_{i}$.

Then we have a point $\hat{x}=\left(\widehat{x}_{i}\right)_{i \in I} \in X$ such that $\widehat{x}_{i} \in T_{i}\left(g_{i}(\hat{x})\right)$ for each $i \in I$.

Proof. Using the similar argument of Theorem 10, we only need to show that the conditions (ii) and (iii) of Theorem 10 are satisfied. By the definition of $\operatorname{GFC}\left(R_{i}\left(S_{i}\left(z_{i}\right)\right)\right)$, we know that $\operatorname{GFC}\left(R_{i}\left(S_{i}\left(z_{i}\right)\right)\right)$ is a GFC-subspace of $X_{i}$ with respect to $S_{i}\left(z_{i}\right)$. It then follows from condition (ii) that $T_{i}\left(z_{i}\right)$ is a GFC-subspace of $X_{i}$ with respect to $S_{i}\left(z_{i}\right)$, which means that the condition (i) of Theorem 10 holds. It remains to deal with condition (iii). If $Z_{i}$ is noncompact, by (iii), we get

$$
B_{i}=\bigcap_{y_{i} \in Y_{i}^{0}}\left(\operatorname{cint} S_{i}^{-1}\left(y_{i}\right)\right)^{c}=Y_{i} \backslash \bigcup_{y_{i} \in Y_{i}^{0}} \operatorname{cint} S_{i}^{-1}\left(y_{i}\right) \subset D_{i} .
$$

Clearly $B_{i}$ is a closed subset of compact set $D_{i}$. If $B_{i}$ is nonempty, then $B_{i}$ is compact in $Z_{i}$. This means that the condition (iii) of Theorem 10 is satisfied. So the statement of Theorem 11 follows immediately from Theorem 10 .

Remark 12. (a) Let $V_{j}$ be a topological spaces for each $j \in$ $J$. By Lemma 5, if using assumption (i) in Lemma 5 for assumption (i) in Theorems 9 and 10, then the statements of Theorems 9 and 10 are still true.

(b) The results of Theorems 10 and 11 generalize Theorem 2.1 of Lan and Webb [12], Theorem 1 of Ansari and Yao [13], Theorem 2.2 of Ding and Park [14], Theorem 3.1 of Ding and Park [15], and Theorem 3.1 of Lin and Ansari [16] and Ding [2] to GFC-spaces.

\section{Coincidence Theorems for Two Families of Set-Valued Mappings}

Theorem 13. Let $\left(X_{i}, Y_{i},\left\{\varphi_{N_{i}}\right\}\right)$ and $\left(U_{j}, V_{j},\left\{\varphi_{N_{j}}\right\}\right)$ be GFCspaces for any $i \in I$ and $j \in J$. Assume that $X=\prod_{i \in I} X_{i}$ and $U=\prod_{i \in J} U_{j}$. Let $S_{i}: U \rightarrow 2^{Y_{i}}, T_{i}: U \rightarrow 2^{X_{i}}, F_{j}: X \rightarrow 2^{V_{j}}$, and $G_{j}: X \rightarrow 2^{U_{j}}$ be set-valued mappings satisfying the following conditions:

(i) for any compact subset $A$ of $X, A=\bigcup_{v_{j} \in V_{j}}\left(\operatorname{cint} F_{j}^{-1}\right.$ $\left.\left(v_{j}\right) \cap A\right)$;

(ii) for any $x \in X, G_{j}(x)$ is a GFC-subspace of $U_{j}$ with respect to $F_{j}(x)$;

(iii) there exists a nonempty subset of $Y_{i}^{0}$ of $Y_{i}$ such that the set $D_{i}=\bigcap_{y_{i} \in Y_{i}^{0}}\left(\operatorname{cint} S_{i}^{-1}\left(y_{i}\right)\right)^{c}$ is empty or compact in $U$ and for each $N_{i} \in\left\langle Y_{i}\right\rangle$, there is a compact GFCsubspace $K_{N_{i}}$ of $X_{i}$ with respect to $L_{N_{i}}$ containing $Y_{i}^{0} U$ $N_{i}$;

(iv) for any compact subset $B$ of $U, B=\bigcup_{y_{i} \in Y_{i}}\left(\operatorname{cint} S_{i}^{-1}\left(y_{i}\right) \cap\right.$ $B)$;

(v) for any $u \in U, T_{i}(u)$ is a GFC-subspace of $X_{i}$ with respect to $S_{i}(u)$.

Then one has $\hat{x}=\left(\widehat{x}_{i}\right)_{i \in I} \in X$ and $\widehat{u}=\left(\widehat{u}_{j}\right)_{j \in J} \in U$ such that $\widehat{x}_{i} \in T_{i}(\widehat{u})$ and $\widehat{u}_{j} \in G_{j}(\widehat{x})$ for any $i \in I$ and $j \in J$.

Proof. For any given $i \in I$, if $D_{i}$ is empty in (iii), it is easy to see that

$$
U=U \backslash D_{i}=U \backslash \bigcap_{y_{i} \in Y_{i}^{0}}\left(\operatorname{cint} S_{i}^{-1}\left(y_{i}\right)\right)^{c}=\bigcup_{y_{i} \in Y_{i}^{0}} \operatorname{cint} S_{i}^{-1}\left(y_{i}\right) .
$$

If $D_{i}$ is nonempty compact set in $U$, by (iv), we know that

$$
D_{i}=\bigcup_{y_{i} \in Y_{i}}\left(\operatorname{cint} S_{i}^{-1}\left(y_{i}\right) \cap D_{i}\right) \subset \bigcup_{y_{i} \in Y_{i}} \operatorname{cint} S_{i}^{-1}\left(y_{i}\right) \text {. }
$$

Note that $D_{i}$ is compact. Then we can find a finite set $N_{i} \in$ $\left\langle Y_{i}\right\rangle$ such that

$$
D_{i}=\bigcap_{y_{i} \in Y_{i}^{0}}\left(\operatorname{cint} S_{i}^{-1}\left(y_{i}\right)\right)^{c} \subset \bigcup_{y_{i} \in N_{i}} \operatorname{cint} S_{i}^{-1}\left(y_{i}\right) .
$$

It then follows from (16) and (18) that if either $D_{i}$ is empty or compact in $U$, we get

$$
U=\bigcup_{y_{i} \in Y_{i}^{0}} \operatorname{cint} S_{i}^{-1}\left(y_{i}\right) \cup\left(\bigcup_{y_{i} \in N_{i}} \operatorname{cint} S_{i}^{-1}\left(y_{i}\right)\right) .
$$


Furthermore, by (iii), there exists a compact GFCsubspace $K_{N_{i}}$ of $X_{i}$ with respect to $L_{N_{i}}$ containing $Y_{i}^{0} \cup N_{i}$. So by (19), we get

$$
U=\bigcup_{y_{i} \in L_{N_{i}}}\left(\operatorname{cint} S_{i}^{-1}\left(y_{i}\right)\right)
$$

Assume that $K_{N}=\prod_{i \in I} K_{N_{i}}, L_{N}=\prod_{i \in I} L_{N_{i}}$, and $\varphi_{N}=$ $\prod_{i \in I} \varphi_{N_{i}}$. It then follows from Lemma 6 that $K_{N}$ is a compact GFC-subspace of $X$ with respect to $L_{N}$. So $\left(K_{N}, L_{N},\left\{\varphi_{N}\right\}\right)$ is a compact GFC-space. We consider the restrictions $\left.F_{j}\right|_{K_{N}}$ and $\left.G_{j}\right|_{K_{N}}$ of $F_{j}$ and $G_{j}$ on $K_{N}$, respectively, for each $j \in J$. Then by condition (ii), we have $\left.G_{j}\right|_{K_{N}}(x)$ as a GFC-subspace of $U_{j}$ with respect to $\left.F_{j}\right|_{K_{N}}(x)$ for any $x \in X$. From condition (i), we obtain

$$
K_{N}=\bigcup_{v_{j} \in V_{j}}\left(\operatorname{cint} F_{j}^{-1}\left(v_{j}\right) \cap K_{N}\right)=\left.\bigcup_{v_{j} \in V_{j}} \operatorname{cint} F_{j}^{-1}\right|_{K_{N}}\left(v_{j}\right) .
$$

Moreover, by Theorem 7, there exists a continuous selection $g_{j}: K_{N} \rightarrow U_{j}$ of $\left.G_{j}\right|_{K_{N}}$ for any $j \in J$. Let $g: K_{N} \rightarrow U$ be a mapping with $g(x)=\prod_{j \in J} g_{j}(x)$ for any $x \in L_{N}$. Clearly $g$ is a continuous mapping. Define two set-valued mappings $P_{i}: K_{N} \rightarrow 2^{L_{N_{i}}}$ and $Q_{i}: K_{N} \rightarrow 2^{K_{N_{i}}}$ by

$$
P_{i}(x)=S_{i}(g(x)) \cap L_{N_{i}}, \quad Q_{i}(x)=T_{i}(g(x)) \cap K_{N_{i}} .
$$

Since $K_{N_{i}}$ is a compact GFC-subspace of $X_{i}$ relative to $L_{N_{i}}$, by (v), we know that $Q_{i}(x)$ is also a compact GFCsubspace of $X_{i}$ with respect to $P_{i}(x)$.

Now we claim that $K_{N}=\bigcup_{y_{i} \in L_{N_{i}}} \operatorname{cint} P_{i}^{-1}\left(y_{i}\right)$. For any given $y_{i} \in L_{N_{i}}$, we deduce that

$$
\begin{aligned}
P_{i}^{-1}\left(y_{i}\right) & \\
& =\left\{x \in K_{N}: y_{i} \in P_{i}(x)\right\} \\
& =\left\{x \in K_{N}: y_{i} \in S_{i}(g(x)) \cap L_{N_{i}}\right\} \\
& =\left\{x \in K_{N}: y_{i} \in S_{i}(g(x))\right\} \\
& =\left\{x \in K_{N}: x \in g^{-1}\left(S_{i}^{-1}\left(y_{i}\right)\right)\right\} \\
& =g^{-1}\left(S_{i}^{-1}\left(y_{i}\right)\right) .
\end{aligned}
$$

Then by (20) we obtain

$$
g\left(K_{N}\right) \subset U=\bigcup_{y_{i} \in L_{N_{i}}}\left(\operatorname{cint} S_{i}^{-1}\left(y_{i}\right)\right) .
$$

It then follows form (23), (24), and the continuity of $g$ that

$$
\begin{aligned}
K_{N} & \subset g^{-1}\left(\bigcup_{y_{i} \in L_{N_{i}}} \operatorname{cint} S_{i}^{-1}\left(y_{i}\right)\right) \\
& =\bigcup_{y_{i} \in L_{N_{i}}} g^{-1}\left(\operatorname{cint} S_{i}^{-1}\left(y_{i}\right)\right) \\
& =\bigcup_{y_{i} \in L_{N_{i}}}\left(\operatorname{cint} P_{i}^{-1}\left(y_{i}\right)\right) \subset K_{N} .
\end{aligned}
$$

Hence $K_{N}=\bigcup_{y_{i} \in L_{N_{i}}} \operatorname{cint} P_{i}^{-1}\left(y_{i}\right)$. So the claim is proved.

Note that $Q_{i}(x)$ is a compact GFC-subspace of $X_{i}$ relative to $P_{i}(x)$. So by the above claim and Theorem 9 , we know that there exists a point $\widehat{x} \in K_{N} \subset X$ such that $\widehat{x}_{i} \in Q_{i}(\hat{x})=$ $T_{i}(g(\hat{x})) \cap K_{N_{i}} \subset T_{i}(g(\hat{x}))$ for any given $i \in I$. Assume that $\widehat{u}=g(\widehat{x})=\prod_{j \in J} g_{j}(\widehat{x})$. Thus we derive that there exist $\widehat{x} \in X$ and $\widehat{u} \in U$ such that $\widehat{x}_{i} \in T_{i}(\widehat{u})$ and $\widehat{u}_{j} \in G_{j}(\widehat{x})$ for any $i \in I$ and $j \in J$, which implies that Theorem 13 is true.

Theorem 14. Let $\left(X_{i}, Y_{i},\left\{\varphi_{N_{i}}\right\}\right)$ and $\left(U_{j}, V_{j},\left\{\varphi_{N_{j}}\right\}\right)$ be GFCspaces for any $i \in I$ and $j \in J$. Assume that $X=\prod_{i \in I} X_{i}$ and $U=\prod_{i \in J} U_{j}$. Let $S_{i}: U \rightarrow 2^{Y_{i}}, T_{i}: U \rightarrow 2^{X_{i}}, R_{i}: Y_{i} \rightarrow 2^{X_{i}}$, $F_{j}: X \rightarrow 2^{V_{j}}, G_{j}: X \rightarrow 2^{U_{j}}$, and $H_{j}: V_{j} \rightarrow 2^{U_{j}}$ be set-valued mappings for each $i \in I$ and $j \in J$. If the following conditions hold:

(i) for any compact subset $A$ of $X, A=\bigcup_{v_{j} \in V_{j}}\left(\operatorname{cint} F_{j}^{-1}\right.$ $\left.\left(v_{j}\right) \cap A\right)$,

(ii) for any $x \in X, G F C\left(H_{j}\left(F_{j}(x)\right)\right) \subset G_{j}(x)$,

(iii) if $U$ is not compact, then there exists a nonempty subset of $Y_{i}^{0}$ of $Y_{i}$ as well as a compact subset $K$ of $U$ such that $U \backslash K \subset \bigcup_{y_{i} \in Y_{i}^{0}} \operatorname{cint} S_{i}^{-1}\left(y_{i}\right)$ and for each $N_{i} \in\left\langle Y_{i}\right\rangle$, there is a compact GFC-subspace $K_{N_{i}}$ of $X_{i}$ with respect to $L_{N_{i}}$ containing $Y_{i}^{0} \cup N_{i}$,

(iv) for any compact subset $B$ of $U, B=\bigcup_{v_{j} \in V_{j}}\left(\operatorname{cint} F_{j}^{-1}\right.$ $\left.\left(v_{j}\right) \cap B\right)$,

(v) for any $u \in U, \operatorname{GFC}\left(R_{i}\left(S_{i}(u)\right)\right) \subset T_{i}(u)$,

then there exist $\widehat{x}=\left(\widehat{x}_{i}\right)_{i \in I} \in X$ and $\widehat{u}=\left(\widehat{u}_{j}\right)_{j \in J} \in U$ such that $\widehat{x}_{i} \in T_{i}(\widehat{u})$ and $\widehat{u}_{j} \in G_{j}(\widehat{x})$ for any $i \in I$ and $j \in J$.

Proof. By the definition of the GFC-hull with respect to $F_{j}(x)$ and condition (ii), it is easy to see that $G_{j}(x)$ is a GFCsubspace of $U_{j}$ with respect to $F_{j}(x)$ for any $x \in X$ and $j \in J$. Similarly, by (v), we obtain that $T_{i}(u)$ is a GFC-subspace of $X_{i}$ with respect to $S_{i}(u)$ for any $u \in U$ and $i \in I$. Then conditions (ii) and (v) of Theorem 13 hold. On the other hand, by (iii), if $U$ is not compact, we know that

$$
D=\bigcap_{y_{i} \in Y_{i}^{0}}\left(\operatorname{cint} S_{i}^{-1}\left(y_{i}\right)\right)^{c}=U \backslash \bigcup_{y_{i} \in Y_{i}^{0}} \operatorname{cint} S_{i}^{-1}\left(y_{i}\right) \subset K .
$$

If $D$ is nonempty, then $D$ is a closed subset of compact set $K$. This implies that $D$ is compact in $U$. So condition (iii) tells us condition (iii) of Theorem 13 holds. Then the statement of Theorem 14 follows immediately from Theorem 13.

Remark 15. Theorems 13 and 14 improve Theorem 9 of Yu and Lin [17], Theorem 3.3 of Lin and Ansari [16], and Theorems 3.1 and 3.2 of Ding [2] to GFC-spaces without any convexity structure.

\section{Applications}

In the current section, we will give some applications of our theorems. 
Theorem 16. Let $\left(X_{i}, Y_{i},\left\{\varphi_{N_{i}}\right\}\right)$ be a GFC-space and $Z_{i}$ a topological space for each $i \in I$. Assume that $A_{i}$ and $B_{i}$ are a subset of $Y_{i} \times Z_{i}$ and $X_{i} \times Z_{i}$, respectively. Let $X=\prod_{i \in I} X_{i}$ and for each $i \in I$, let $g_{i}: X \rightarrow Z_{i}$ be a continuous mapping and $R_{i}: Y_{i} \rightarrow 2^{X_{i}}$ a set-valued mapping satisfying the following conditions:

(i) for any compact subset $D_{i}$ of $Z_{i}, D_{i}=\bigcup_{y_{i} \in Y_{i}}\left(\operatorname{cint}\left\{z_{i} \epsilon\right.\right.$ $\left.\left.Z_{i}:\left(y_{i}, z_{i}\right) \in A_{i}\right\} \cap D_{i}\right)$;

(ii) for any $z_{i} \in Z_{i}$, GFC $\left(R_{i}\left(\left\{y_{i} \in Y_{i}:\left(y_{i}, z_{i}\right) \in A_{i}\right\}\right)\right) \subset$ $\left\{x_{i} \in X_{i}:\left(x_{i}, z_{i}\right) \in B_{i}\right\}$

(iii) if $Z_{i}$ is not compact, then there exists a nonempty subset of $Y_{i}^{0}$ of $Y_{i}$ such that a compact subset $D_{i}$ of $Z_{i}$ such that $Z_{i} \backslash D_{i} \subset \bigcup_{y_{i} \in Y_{i}^{0}} \operatorname{cint}\left\{z_{i} \in Z_{i}:\left(y_{i}, z_{i}\right) \in A_{i}\right\}$ and for each $N_{i} \in\left\langle Y_{i}\right\rangle$, there is a compact GFC-subspace of $X_{i}$ with respect to $L_{N_{i}}$ containing $Y_{i}^{0} \cup N_{i}$.

Then we have a point $\widehat{x}=\left(\widehat{x}_{i}\right)_{i \in I} \in X$ such that $\left(\widehat{x}_{i}, g_{i}(\widehat{x})\right) \in B_{i}$ for each $i \in I$.

Proof. For any given $i \in I$, we define two set-valued mappings $S_{i}: Z_{i} \rightarrow 2^{Y_{i}}$ and $T_{i}: Z_{i} \rightarrow 2^{X_{i}}$ by

$$
\begin{aligned}
& S_{i}\left(z_{i}\right)=\left\{y_{i} \in Y_{i}:\left(y_{i}, z_{i}\right) \in A_{i}\right\}, \\
& T_{i}\left(z_{i}\right)=\left\{x_{i} \in X_{i}:\left(x_{i}, z_{i}\right) \in B_{i}\right\}
\end{aligned}
$$

for all $z_{i} \in Z_{i}$. It then follows from assumptions (i)-(iii) and Theorem 11 that for any $i \in I$, there exists a point $\widehat{x}=\left(\widehat{x}_{i}\right)_{i \in I} \in$ $X$ such that $\widehat{x}_{i} \in T_{i}\left(g_{i}(\widehat{x})\right)$, which implies that $\left(\widehat{x}_{i}, g_{i}(\widehat{x})\right) \in B_{i}$. So Theorem 16 is true.

Corollary 17. For each $i \in I$, let $\left(X_{i}, Y_{i},\left\{\varphi_{N_{i}}\right\}\right)$ be a GFC-space, $R_{i}: Y_{i} \rightarrow 2^{X_{i}}$ a set-valued mapping, $X=\prod_{i \in I} X_{i}$, and $X^{i}=$ $\prod_{j \in I, j \neq i} X_{j}$. Assume that $\left\{A_{i}\right\}_{i \in I}$ and $\left\{B_{i}\right\}_{i \in I}$ are the families of subsets of $Y_{i} \times X^{i}$ and $X_{i} \times X^{i}$, respectively. If the following conditions hold:

(i) for any compact subset $D^{i}$ of $X^{i}, D^{i}=\bigcup_{y_{i} \in Y_{i}}\left(\operatorname{cint}\left\{z_{i} \epsilon\right.\right.$ $\left.\left.Z_{i}:\left(y_{i}, x^{i}\right) \in A_{i}\right\} \cap D^{i}\right)$,

(ii) for all $x^{i} \in X^{i}, \operatorname{GFC}\left(R_{i}\left(\left\{y_{i} \in Y_{i}:\left(y_{i}, x^{i}\right) \in A_{i}\right\}\right)\right) \subset$ $\left\{x_{i} \in X_{i}:\left(x_{i}, x^{i}\right) \in B_{i}\right\}$,

(iii) if $X^{i}$ is not compact, then there exists a nonempty subset of $Y_{i}^{0}$ of $Y_{i}$ such that a compact subset $D^{i}$ of $X^{i}$ such that $X^{i} \backslash D^{i} \subset \bigcup_{y_{i} \in Y_{i}^{0}} \operatorname{cint}\left\{x^{i} \in X^{i}:\left(y_{i}, z_{i}\right) \in A_{i}\right\}$ and for each $N_{i} \in\left\langle Y_{i}\right\rangle$, there is a compact GFC-subspace of $X_{i}$ relative to $L_{N_{i}}$ containing $Y_{i}^{0} \cup N_{i}$,

then $\bigcap_{i \in I} B_{i} \neq \emptyset$.

Proof. Define a mapping $g_{i}: X \rightarrow X^{i}$ as the projection of $X$ onto $X^{i}=\prod_{j \in I, j \neq i} X_{j}$. Clearly $g_{i}$ is a continuous mapping. Using Theorem 16 with $Z_{i}=X^{i}$ and $D_{i}=D^{i}$, we find that there exists a point $\hat{x}=\left(\widehat{x}_{i}\right)_{i \in I} \in X$ such that $\left(\widehat{x}_{i}, g_{i}(\hat{x})\right) \in B_{i}$ for any $i \in I$, which implies that $\widehat{x}=\left(\widehat{x}_{i}, g_{i}(\widehat{x})\right) \in \bigcap_{i \in I} B_{i}$. Hence $\bigcap_{i \in I} B_{i} \neq \emptyset$.
In the rest of this section, for each $i \in I$ and $j \in J$, we always let $Z_{i}$ and $W_{i}$ be two nonempty set and $\left(X_{i}, Y_{i},\left\{\varphi_{N_{i}}\right\}\right)$ and $\left(U_{j}, V_{j},\left\{\varphi_{N_{j}}\right\}\right)$ be two GFC-spaces. Assume that $X=$ $\prod_{i \in I} X_{i}$ and $U=\prod_{i \in J} U_{j}$. For any $i \in I$ and $j \in J$, let $R_{i}: Y_{i} \rightarrow$ $2^{X_{i}}, H_{j}: V_{j} \rightarrow 2^{U_{j}}, C_{j}: X \rightarrow 2^{Z_{j}}, D_{i}: U \rightarrow 2^{W_{i}}, B_{j}:$ $X \times V_{j} \rightarrow 2^{Z_{j}}, A_{j}: X \times U_{j} \rightarrow 2^{Z_{j}}, Q_{i}: Y_{i} \times U \rightarrow 2^{W_{i}}$, and $P_{i}: X_{i} \times U \rightarrow 2^{W_{i}}$ be set-valued mappings. Then we have the follows results.

Theorem 18. Suppose that the following conditions hold:

(i) for any compact subset $A$ of $X, A=\bigcup_{v_{j} \in V_{j}}(\operatorname{cint}\{x \in$ $\left.\left.X: B_{j}\left(x, v_{j}\right) \subset C_{j}(x)\right\} \cap A\right) ;$

(ii) for any $x \in X, \operatorname{GFC}\left(H_{j}\left(\left\{v_{j} \in V_{j}: B_{j}\left(x, v_{j}\right) \subset\right.\right.\right.$ $\left.\left.\left.C_{j}(x)\right\}\right)\right) \subset\left\{u_{j} \in U_{j}: A_{j}\left(x, u_{j}\right) \subset C_{j}(x)\right\} ;$

(iii) if $U$ is not compact, then there exists a nonempty subset of $Y_{i}^{0}$ of $Y_{i}$ such that a compact subset $K$ of $U$ such that $U \backslash K \subset \bigcup_{y_{i} \in Y_{i}^{0}} \operatorname{cint}\left\{u \in U: Q_{i}\left(y_{i}, u\right) \subset D_{i}(u)\right\}$ and for each $N_{i} \in\left\langle Y_{i}\right\rangle$, there is a compact GFC-subspace of $X_{i}$ with respect to $L_{N_{i}}$ containing $Y_{i}^{0} \cup N_{i}$;

(iv) for any compact subset $B$ of $U, B=\bigcup_{y_{i} \in Y_{i}}\left(\operatorname{cint}\left\{y_{i} \in\right.\right.$ $\left.\left.Y_{i}: Q_{i}\left(y_{i}, u\right) \subset D_{i}(u)\right\} \cap B\right) ;$

(v) for any $u \in U, G F C\left(R_{i}\left(\left\{y_{i} \in Y_{i}: Q_{i}\left(y_{i}, u\right) \subset\right.\right.\right.$ $\left.\left.\left.D_{i}(u)\right\}\right)\right) \subset\left\{x_{i} \in X_{i}: P_{i}\left(x_{i}, u\right) \subset D_{i}(u)\right\}$.

Then there exist $(\widehat{x}, \widehat{u}) \in X \times U$ and $\widehat{u}=\left(\widehat{u}_{j}\right)_{j \in J} \in U$ such that $A_{j}\left(\widehat{x}, \widehat{u}_{j}\right) \subset C_{j}(\widehat{x})$ and $P_{i}\left(\widehat{x}_{i}, \widehat{u}\right) \subset D_{i}(\widehat{u})$ for each $i \in I$ and $j \in J$.

Proof. Assume that $S_{i}: U \rightarrow 2^{Y_{i}}, T_{i}: U \rightarrow 2^{X_{i}}, F_{j}: X \rightarrow$ $2^{V_{j}}$, and $G_{j}: X \rightarrow 2^{U_{j}}$ are set-valued mappings as follows:

$$
\begin{aligned}
& S_{i}(u)=\left\{y_{i} \in Y_{i}: Q_{i}\left(y_{i}, u\right) \subset D_{i}(u)\right\}, \\
& T_{i}(u)=\left\{x_{i} \in X_{i}: P_{i}\left(x_{i}, u\right) \subset D_{i}(u)\right\} \quad \forall u \in U, \\
& F_{j}(x)=\left\{v_{j} \in V_{j}: B_{j}\left(x, v_{j}\right) \subset C_{j}(x)\right\}, \\
& G_{j}(x)=\left\{u_{j} \in U_{j}: A_{j}\left(x, u_{j}\right) \subset C_{j}(x)\right\} \quad \forall x \in X .
\end{aligned}
$$

It then follows from Theorem 14 that there exist $\hat{x} \in X$ and $\widehat{u} \in U$ such that $\widehat{x}_{i} \in T_{i}(\widehat{u})$ and $\widehat{u}_{j} \in G_{j}(\widehat{x})$ for any $i \in I$ and $j \in J$. By the above definition, we obtain that $A_{j}\left(\widehat{x}, \widehat{u}_{j}\right) \subset$ $C_{j}(\widehat{x})$ and $P_{i}\left(\widehat{x}_{i}, \widehat{u}\right) \subset D_{i}(\widehat{u})$ for any $i \in I$ and $j \in J$. This completes the proof.

Theorem 19. For each $i \in I$ and $j \in J$, let $Y_{i}$ and $V_{j}$ be topological spaces. Suppose that the following conditions hold:

(i) for each $x \in X$, the set $\left\{v_{j} \in V_{j}: B_{j}\left(x, v_{j}\right) \subset C_{j}(x)\right\}$ is nonempty and $B_{j}\left(x, v_{j}\right)$ is transfer compactly upper semicontinuous in $x$ with respect to $C_{j}$;

(ii) for any $x \in X, \operatorname{GFC}\left(H_{j}\left(\left\{v_{j} \in V_{j}: B_{j}\left(x, v_{j}\right) \subset\right.\right.\right.$ $\left.\left.\left.C_{j}(x)\right\}\right)\right) \subset\left\{u_{j} \in U_{j}: A_{j}\left(x, u_{j}\right) \subset C_{j}(x)\right\}$;

(iii) if $U$ is not compact, then there exists a nonempty subset of $Y_{i}^{0}$ of $Y_{i}$ as well as a compact subset $K$ of $U$ such that 
$U \backslash K \subset \bigcup_{y_{i} \in Y_{i}^{0}} \operatorname{cint}\left\{u \in U: Q_{i}\left(y_{i}, u\right) \subset D_{i}(u)\right\}$ and for each $N_{i} \in\left\langle Y_{i}\right\rangle$, there is a compact GFC-subspace of $X_{i}$ with respect to $L_{N_{i}}$ containing $Y_{i}^{0} \cup N_{i}$;

(iv) for each $u \in U$, the set $\left\{y_{i} \in Y_{i}: Q_{i}\left(y_{i}, u\right) \subset D_{i}(u)\right\}$ is nonempty and $Q_{i}\left(y_{i}, u\right)$ is transfer compactly upper semicontinuous in $u$ with respect to $D_{i}$;

(v) for any $u \in U, \operatorname{GFC}\left(R_{i}\left(\left\{y_{i} \in Y_{i}: Q_{i}\left(y_{i}, u\right) \subset D_{i}(u)\right\}\right)\right)$ $\subset\left\{x_{i} \in X_{i}: P_{i}\left(x_{i}, u\right) \subset D_{i}(u)\right\}$.

Then there exist $(\widehat{x}, \widehat{u}) \in X \times U$ and $\widehat{u}=\left(\widehat{u}_{j}\right)_{j \in J} \in U$ such that $A_{j}\left(\widehat{x}, \widehat{u}_{j}\right) \subset C_{j}(\widehat{x})$ and $P_{i}\left(\widehat{x}_{i}, \widehat{u}\right) \subset D_{i}(\widehat{u})$ for all $i \in I$ and $j \in J$.

Proof. Let $S_{i}, T_{i}, F_{j}$, and $G_{j}$ be set-valued mappings as defined in the proof of Theorem 18. Using the same argument of Theorem 18, we only need to show that the assumptions (i) and (iv) of Theorem 18 hold. From assumption (i) and Lemma 4 , we know that for any $x \in X, F_{j}^{-1}$ is transfer compactly open-valued. Thus Lemma 5 tells us that the assumption (i) of Theorem 18 holds. In a similar way, from (iv) and Lemma 4, we obtain that the assumption (iv) of Theorem 18 is satisfied. So the statement of Theorem 19 follows immediately from Theorem 18 .

\section{Conflict of Interests}

The author declares that there is no conflict of interests regarding the publication of this paper.

\section{Acknowledgment}

The author would like to thank the anonymous referees for careful reading of the paper and for helpful comments and suggestions which improved its presentation.

\section{References}

[1] X. P. Ding, "Maximal element theorems in product FC-spaces and generalized games," Journal of Mathematical Analysis and Applications, vol. 305, no. 1, pp. 29-42, 2005.

[2] X. P. Ding, "Collective fixed points and a system of coincidence theorems in product FC-spaces and applications," Nonlinear Analysis: Theory, Methods and Applications, vol. 66, no. 11, pp. 2604-2617, 2007.

[3] X. P. Ding and T. M. Ding, "KKM type theorems and generalized vector equilibrium problems in noncompact FC-spaces," Journal of Mathematical Analysis and Applications, vol. 331, no. 2, pp. 1230-1245, 2007.

[4] G. Tang, Q. Zhang, and C. Cheng, "W-G-F-KKM mapping, intersection theorems and minimax inequalities in FC-space," Journal of Mathematical Analysis and Applications, vol. 334, no. 2, pp. 1481-1491, 2007.

[5] M. Fang and N. Huang, "KKM type theorems with applications to generalized vector equilibrium problems in FC-spaces," Nonlinear Analysis: Theory, Methods and Applications, vol. 67, no. 3, pp. 809-817, 2007.

[6] P. Q. Khanh and N. H. Quan, "Intersection theorems coincidence theorems and maximal-element theorems in GFCspaces," Optimization, vol. 59, pp. 29-42, 2010.
[7] P. Q. Khanh, N. H. Quan, and J. Yao, "Generalized KKM-type theorems in GFC-spaces and applications," Nonlinear Analysis: Theory, Methods and Applications, vol. 71, no. 3-4, pp. 1227-1234, 2009.

[8] X. P. Ding, "Coincidence theorems in topological spaces and their applications," Applied Mathematics Letters, vol. 12, no. 7, pp. 99-105, 1999.

[9] X. P. Ding and J. Y. Park, "Generalized vector equilibrium problems in generalized convex spaces," Journal of Optimization Theory and Applications, vol. 120, no. 2, pp. 327-353, 2004.

[10] E. Tarafdar, "Fixed point theorems in $\mathrm{H}$-spaces and equilibrium points of abstract economies," Journal of the Australian Mathematical Society. Series A, vol. 53, no. 1, pp. 252-260, 1992.

[11] F. E. Browder, "Coincidence theorems, minimax theorems, and variational inequalities," Contemporary Mathematics, vol. 26, pp. 67-80, 1984.

[12] K. Lan and J. Webb, "New fixed point theorems for a family of mappings and applications to problems on sets with convex sections," Proceedings of the American Mathematical Society, vol. 126, no. 4, pp. 1127-1132, 1998.

[13] Q. H. Ansari and J. Yao, "A fixed point theorem and its applications to a system of variational inequalities," Bulletin of the Australian Mathematical Society, vol. 59, no. 3, pp. 433-442, 1999.

[14] X. Ding and J. Park, "New collectively fixed point theorems and applications in G-convex spaces," Applied Mathematics and Mechanics. English Edition, vol. 23, no. 11, pp. 1237-1249, 2002.

[15] X. P. Ding and J. Y. Park, "Collectively fixed-point theorems in noncompact G-convex spaces," Applied Mathematics Letters, vol. 16, no. 3, pp. 137-142, 2003.

[16] L. Lin and Q. H. Ansari, "Collective fixed points and maximal elements with applications to abstract economies," Journal of Mathematical Analysis and Applications, vol. 296, no. 2, pp. 455472, 2004.

[17] Z. Yu and L. Lin, "Continuous selection and fixed point theorems," Nonlinear Analysis: Theory, Methods and Applications, vol. 52, no. 2, pp. 445-455, 2003. 


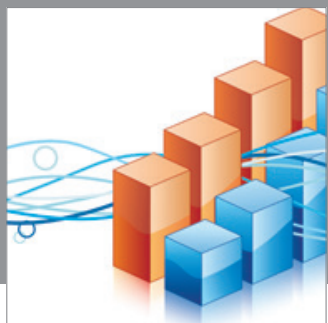

Advances in

Operations Research

mansans

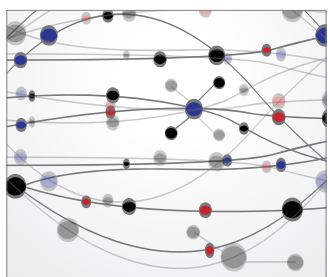

The Scientific World Journal
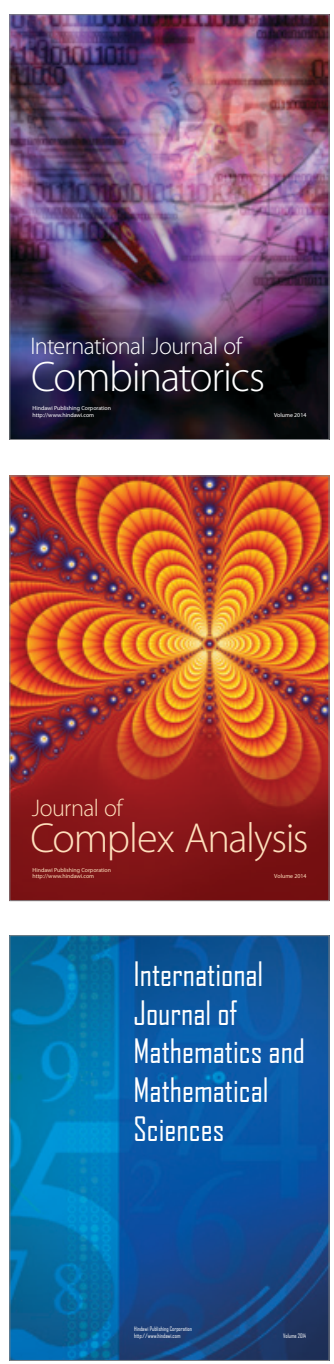
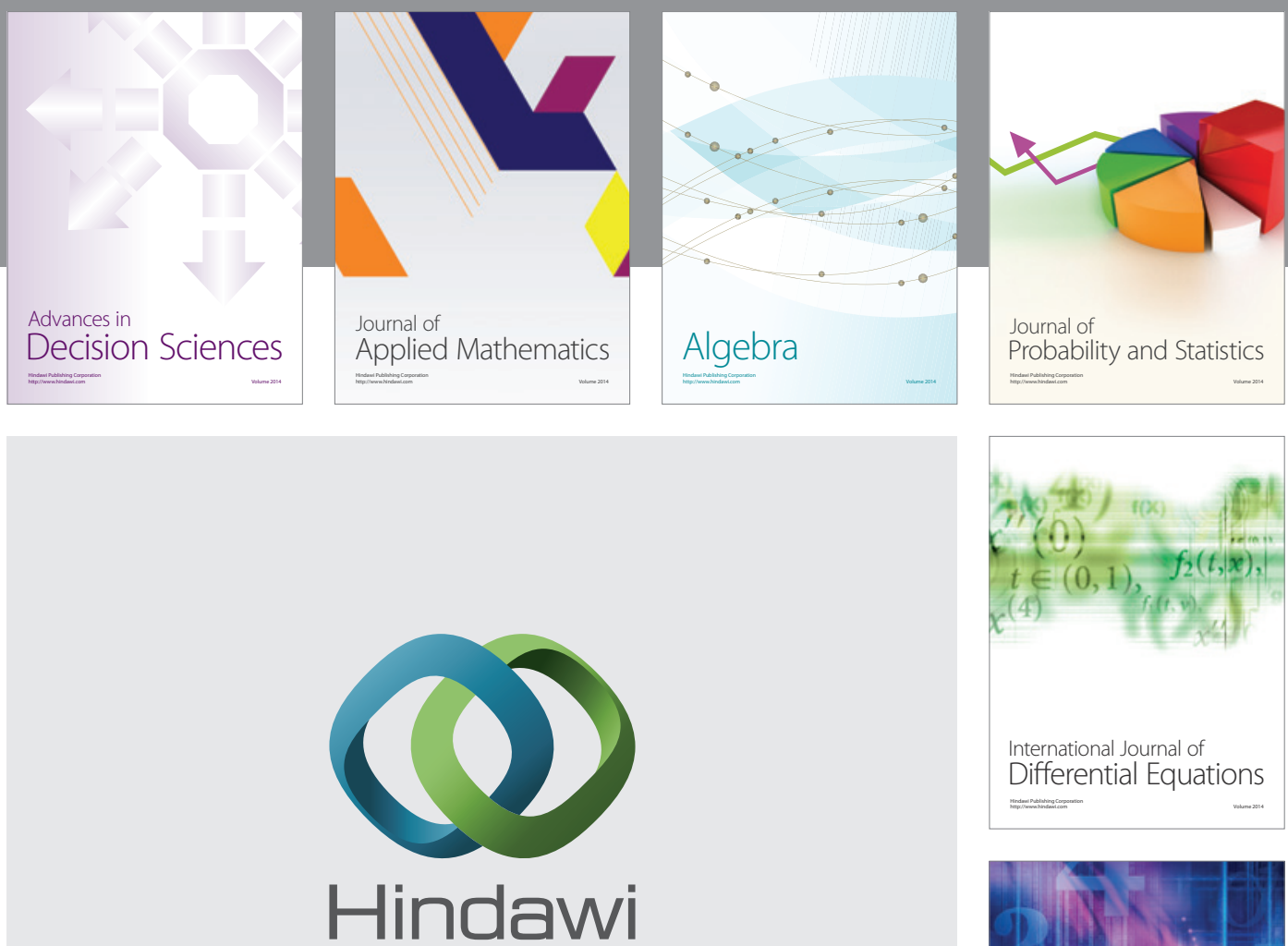

Submit your manuscripts at http://www.hindawi.com
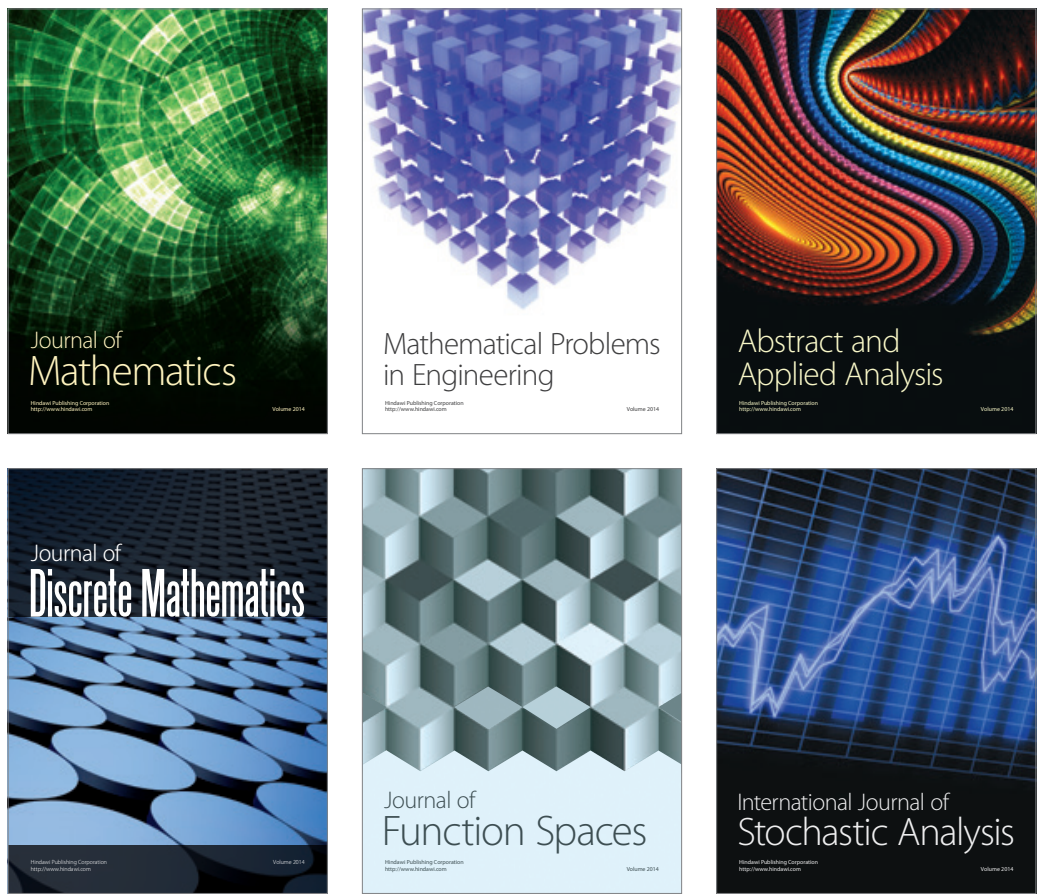

Journal of

Function Spaces

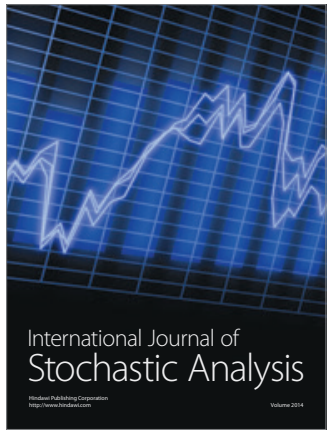

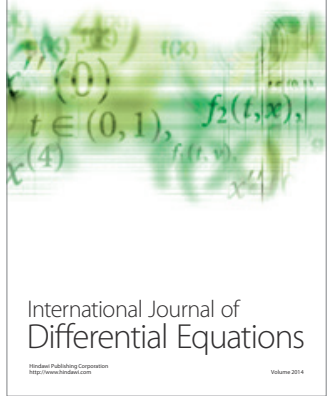
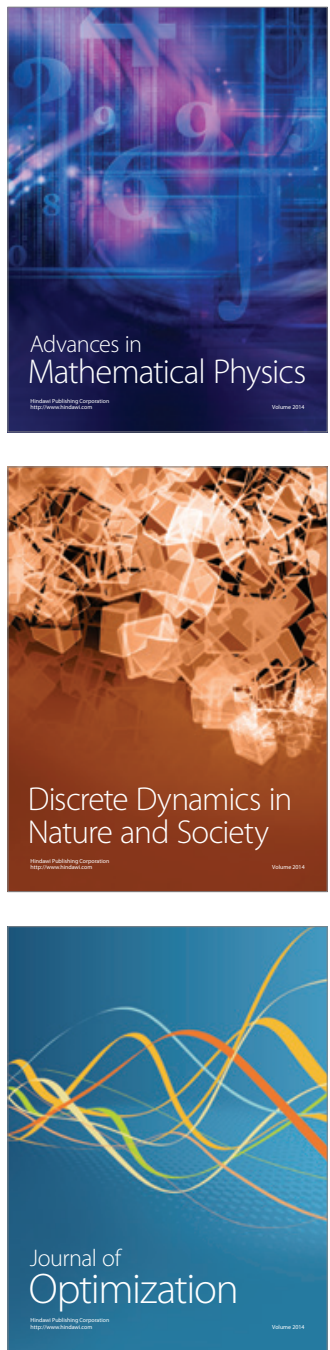